\title{
APROXIMACIÓN METODOLÓGICA AL ANÁLISIS DE LA ESTRUCTURA DE LAS TENDENCIAS DE LLUVIA
}

\author{
José Carlos GONZÁLEZ HIDALGO \\ Departamento de Geografía y Ordenación del Territorio \\ Universidad de Zaragoza
}

Martín De LUIS, José RAVENTÓS, Juan Rafael SÁNCHEZ y Jordi CORTINA

Departamento de Ecología

Universidad de Alicante

\begin{abstract}
Resumen: Los autores desarrollan las técnicas más elementales para detectar cambios en el régimen de precipitación y defienden la tesis de que sin el estudio de la tendencia del cambio de la variabilidad, cualquier predicción basada en el análisis simple del signo de las tendencias o la magnitud del cambio en el volumen puede ocultar errores que lleven a predicciones sumamente equivocadas.
\end{abstract}

Palabras Clave:. Tendencias de lluvia; tendencias de la variabilidad de la lluvia; metodología; test de Spearman.

\begin{abstract}
Authors develop basic techniques to detect change in rainfall regime. It is defended the thesis that any rainfall prediction developed by volume trend analysis without variability trend analysis could be in error.
\end{abstract}

Key words:. Rainfall trend; rainfall trends variability; methodology analysis; Spearman test.

\section{EL CAMBIO GLOBAL Y LAS TENDENCIAS DE LA LLUVIA}

En los últimos años se anuncia un cambio del clima asociado al calentamiento global. En el presente siglo se ha registrado un incremento generalizado de las temperaturas en el 
planeta (FOLLAND et al., 1984; JONES et al., 1986; GATES et al., 1992; HOUGHTON et al., 1996), que ha sido constatado también en la Península Ibérica y el conjunto de la cuenca del Mediterráneo (ESTEBAN-PARRA et al., 1995; OÑATE y POU, 1996; KUTIEL y MAHERAS, 1998; MAHERAS y KOLYVA-MACHERA, 1990).

Sin embargo, los resultados relativos a las tendencias de las precipitaciones son menos concluyentes. A diferencia de los resultados obtenidos con los registros de temperaturas, la evolución en el tiempo de las precipitaciones muestra considerables variaciones tanto en la escala planetaria (BRADLEY et al., 1987; DIAZ et al., 1989), como en la regional (MAHERAS, 1988; HULME, 1992, YU y NEIL, 1993; SUPPIAH y HENESSY, 1998) y la local (RODRIGUEZ-PUEBLA et al., 1998; De LUÍS et al., 1998).

Los resultados propuestos por los Modelos de Circulación General (GCMs) tienen un grado de fiabilidad muy elevado para las predicciones en las escalas planetarias o continentales, pero su precisión disminuye cuando la escala de trabajo aumenta. En el espacio esto ocurre al pasar de la escala zonal a las regionales o locales, y en el tiempo al pasar de las predicciones anuales a las estacionales, mensuales o diarias (REED, 1986; WILSON y MITCHELL, 1987; RIND et al., 1989, GREGORY y MITCHELL, 1995; MEARNS et al., 1996; BARROW et al., 1996; PALUTIKOF et al., 1996; OSBORN y HULME, 1998).

Esta situación explica que una de la necesidades más urgentes de los estudios del cambio climático sea la validación de los resultados de los modelos con datos procedentes de observatorios (HOUGHTON et al., 1990; SALINGER et al., 1995; SUPPIAH y HENNESSY, 1998).

Sin embargo, esta aproximación estadística no permite determinar una relación causa efecto, ni tampoco discernir si los cambios detectados son debidos a la influencia humana (particularmente el calentamiento global) o a la propia variabilidad natural, aunque la coincidencia de ambos resultados (GCMs y estudios basados en registros históricos), podría sugerir que las tendencias observadas se debiesen al calentamiento global al menos en parte (PALUTIKOF et al., 1996). Como mínimo, HULME (1995) y MORON et al., (1995) indican que los registros históricos para estos contrastes deberían ser de al menos de 30 años de duración y a ser posible referidos a periodos normales según convención de WMO.

\section{LA ESTRUCTURA DEL CAMBIO DE LAS VARIABLES CLIMÁTICAS. EL CASO DE LAS PRECIPITACIONES}

La relativa facilidad para detectar el cambio en algunos elementos climáticos por la persistencia del propio elemento (caso de las temperaturas, campos de presión etc), no ocurre en las precipitaciones por tratarse de sucesos discretos (lluvia o no lluvia), porque las lluvias son el elemento del clima más aleatorio y porque es el que estâ sometido a una mayor variabilidad (GROISMAN y LEGATES, 1994). 
Esta variabilidad, además, se presenta como un fenómeno complejo y puede afectar de manera diferente a los volúmenes totales de precipitación, a su distribución mensual o diaria, y a la variabilidad interanual asociada a todos ellos (GORDEN et al, 1992; KATZ y BROWN, 1992; WHETTON et al., 1993; FOWLER y HENNESSY, 1995; GREGORY y MITCHELL, 1995; HENNESSY et al., 1997; HULME y NEW, 1997; MEARNS et al., 1997; McGUFFIE et al.,1999).

Por esta razón los investigadores señalan con insistencia la necesidad de estudiar el cambio tanto en los volúmenes como en la variabilidad, y ambas en diferentes escalas espaciales y temporales, pues estos aspectos son claves para conocer el comportamiento del elemento climático y determinan la posibilidad de realizar predicciones fiables o no en la propia variable (HULME, 1992; SEMENOV y PORTER, 1994; WAYLEN et al., 1996; CORTE-REAL et al, 1998).

\section{El análisis de tendencias}

Por la propia naturaleza de los datos climáticos, para detectar los cambios temporales se emplean dos tipos de métodos. Por un lado los modelos no paramétricos, y por otro los modelos lineales. Aunque ambos métodos son complementarios, no es común que se apliquen ambas técnicas a la vez. Los modelos no paramétricos informan del signo del cambio y su significación, mientras los modelos lineales proporcionan una estimación de la magnitud del cambio (YU y NEIL, 1993; SUPPIAH y HENNESSY, 1998). En este trabajo nos centraremos en la detección del signo del cambio o tendencia, y el signo de la variabilidad del elemento lluvia.

La técnica tradicional del análisis de tendencias es la media móvil, la cual ha sido reconocida como una técnica necesaria para reducir la variabilidad natural de las series (SNEYERS, 1992). El procedimiento permite filtrar parte de la variabilidad entre años, estabiliza las series y detecta señales débiles y tendencias persistentes de larga duración (WHEELER y MARTIN-VIDE, 1992; SALINGER et al., 1995). La técnica ha sido empleada con excelentes resultados en estudios similares en diversas partes del mundo (LOUREIRO y COUTINHO, 1995; KEIM et al., 1995; SUPPIAH y HENNESSY, 1998). Pero el simple suavizado y eliminación de cambios aleatorios no basta para detectar cambios en el tiempo, y se requieren técnicas más complejas.

Probablemente, los modelos estadísticos lineales son la técnica más empleada para buscar relaciones entre dos variables (GREGORY, 1978; LANZANTE, 1996), que en este caso serán el tiempo y el valor de la lluvia o su variabilidad. Sin embargo, las tendencias en series climatológicas raramente son lineales (CLARK y HOSKING, 1986). Además los modelos lineales también tienen sus limitaciones, pues asumen la normalidad y homogeneidad de varianza a lo largo de las series, y con mucha frecuencia están afectados por la presencia de valores extremos u otras formas de no normalidad (LANZANTE, 1996). 
Una posible solución a este problema puede ser emplear técnicas no paramétricas que no se vean afectadas por distribuciones no normales y permitan además analizar la dependencia entre variables, sea aquella lineal o no (DAVIS, 1986; ROSSI et al., 1992). E1 único problema es que estos procedimientos no paramétricos, a pesar de ser ampliamente recomendados (SNEYERS, 1990, 1992), por su propia naturaleza basada en análisis de rangos no permiten conocer la magnitud con se producen los cambios, la cual debe ser explorada mediante procedimientos paramétricos.

Entre las pruebas no paramétricas, la prueba de rangos de Spearman es quizá la más sencilla de aplicar, y está incluida en la mayoría de los paquetes informáticos. Además, cuenta con numerosos casos que avalan su uso en el análisis de tendencias de lluvia (KEIM et al., 1995; LOUREIRO y COUTINHO, 1995; MENDOÇA-LEITE y PEIXOTO, 1996; TÜRKES, 1996; LABAJO et al., 1998; SUPPIAH y HENNESSY, 1998)

\section{La detección del signo del cambio}

En el test de Spearman el rango $y_{i^{\prime}}$ de los elementos $x_{i}$ debe ser determinado en la serie de datos ordenada en sentido creciente. El coeficiente $r_{5}$ es el coeficiente de correlación de la regresión lineal entre la serie de tiempo $(i)$ y la serie $\left(y_{i}\right)$. Este coeficiente puede ser obtenido a partir de la expresión:

$$
r_{s}=1-\left(\frac{6 \sum\left(y_{i}-i\right)^{2}}{n\left(n^{2}-1\right)}\right)
$$

donde $n$ es el número de registros de la serie, o duración en años, e $i$ es la posición de cada elemento en la serie original. La distribución de $r_{s}$ se aproxima a una distribución normal con media cero. Para determinar cuando la hipótesis nula (no existe tendencia) puede ser rechazada o no, es necesaria calcular el valor de probabilidad según:

$$
\alpha=P\left(|u|>\left|u\left(r_{s}\right)\right|\right)
$$

con

$$
u\left(r_{s}\right)=r_{s}(n-1)^{1 / 2}
$$

Si $a<a_{0^{\prime}}$ la hipótesis nula puede ser rechazada para el nivel de significación $a_{0}$. En general el nivel de significación establecido es el 95\%. En el caso, de que una tendencia significativa sea detectada, esta será positiva o negativa dependiendo de si $r_{s}>0$ or $r_{s}<0$.

Finalmente las estimaciones de la magnitud del cambio de la tendencia se pueden obtener de los valores de la pendiente de la regresión lineal calculada por el método de mínimos cuadrados (que no se desarrollará en el presente trabajo). 


\section{La variabilidad y la detección de la tendencia asociada}

La variabilidad de las precipitaciones de un período puede ser descrita globalmente por medio de su coeficiente de variación (véase ANANTHAKRISHNAN y SOMAN, 1989, HULME, 1992, TÜRKES, 1996, RODRIGUEZ-PUEBLA et al, 1998), pues al ser una medida relativa de la serie representa su variabilidad con independencia del valor promedio. Sin embargo el coeficiente de variación es una medida estática, que refleja el valor de la serie de años estudiada, y no informa de la variabilidad entre años.

El estudio de los cambios de la variabilidad entre años se puede realizar utilizando las series de las anomalías de precipitación obtenidas mediante diversos procedimientos (KRAUS, 1977; SRIKANTHAN y STEWART, 1991; SALINGER et al., 1995; TÜRKES, 1996).

Cada anomalía anual se calcula como el valor de desviación entre el valor observado en un determinado año y la media de su serie. En este estudio, al objeto de poder comparar los resultados obtenidos en las diferentes localidades, estas anomalías de precipitación han sido divididas en cada caso por el valor de la lluvia promedio de cada serie, según

$$
A R_{s y}=\frac{R_{s y}-\bar{R}_{s}}{\overline{R_{s}}}
$$

siendo $R_{s y}$ el volumen de lluvia observado en la localidad $s$ en el año $y$; con $\bar{R}_{s}$ la lluvia media de dicha localidad.

Las anomalías, por definición, pueden presentar signos negativos (valores inferiores a la media) y positivos, de modo que para evitar los posibles signos negativos, y puesto que el análisis de la tendencia de la variabilidad trata de detectar simplemente cambios en la regularidad de la lluvia con independencia de su tendencia en volumen, los valores de estas series de anomalías anuales pueden ser manejados como valores absolutos y sometidos al mismo análisis de tendencias que los valores de la variable.

De este modo la variabilidad descrita por la tendencia de la anomalía es un parámetro que describe con mayor precisión las tendencias detectadas en las precipitaciones, por lo que favorece la confección de escenarios futuros en los que realizar los planes de gestión del recurso agua.

\section{EJEMPLOS DE CÁLCULO Y APLICACIÓN A SERIES PLUVIALES}

Los ejemplos reales que se presentan a continuación se obtienen de los trabajos publicados hasta el momento en el marco de los proyectos CICYT, CLI95-1947-CO3-O3 y Generalitat Valenciana GV97-RN-14-2 realizados con las bases de datos diarias del Atlas Climatológico de la Comunidad Valenciana (Pérez Cueva, 1994). Los registros cubren el 
periodo 1950-1990 (41 años) e incluyen en todos los casos el periodo normal 1961-1990, con lo que se asegura la comparación y significación de los resultados con otros observatorios. Los tres casos reales que se presentan han sido seleccionadas al objeto de ilustrar tres situaciones contrastadas. En el texto se incluyen las bases de datos completas de las tres localidades y en el anexo se desarrollan los cálculos pormenorizados en una de ellas.

\section{Caso 1, observatorio de Enguera ( $38^{\circ} 59^{\prime} \mathrm{N}, 0^{\circ} 41^{\prime} \mathrm{W}, 318 \mathrm{~m}$ s.n.m.)}

Los resultados obtenidos son (Tabla 1, Figura 1a y 1b):

- La tendencia de los volúmenes es positiva $(0.616, p=0.000)$, y la tasa de incremento (coeficiente b de la regresión) es de $3.60 \mathrm{~mm} /$ año (Figura 1a)

- La tendencia de la variabilidad obtenida por el método de Spearman es positiva $(0.825, \mathrm{p}=0.000)$ (Figura 1b). La tasa de incremento de esta anomalía medida por el coeficiente de la regresión lineal es de $0.86 \%$ /año.

Caso 2, observatorio de Adzeneta $\left(40^{\circ} 13^{\prime} \mathrm{N}, 1^{\circ} 10^{\prime} \mathrm{W}, 400 \mathrm{~m}\right.$ s.n.m.)

Los resultados indican los siguientes aspectos (Tabla 2 y Figura 2a y 2b):

- La tendencia de los volúmenes obtenida por la prueba de rangos de Spearman es negativa $(-0.744, p=0.000)$, y la tasa a que se produce este descenso es de $-5,55$ $\mathrm{mm} /$ año (Figura 2a).

- La tendencia de la variabilidad no es significativa. El coeficiente de rangos de Spearman aunque tiene signo negativo $(-0.110)$ tiene un bajo valor de probabilidad $(\mathrm{p}=0.534$, Figura 2b).

\section{Caso 3, observatorio de Vila-Real ( $39^{\circ} 56^{\prime} \mathrm{N}, 0^{\circ} 06^{\prime} \mathrm{W}, 42 \mathrm{~m}$ s.n.m.)}

Los resultados se muestran en la Tabla 3 y Figuras $3 a$ y $3 b$.

- La tendencia de los volúmenes es nula. El valor del coeficiente -0.012 no es significativo ( $\mathrm{p}=0.946$, Figura $3 \mathrm{a}$ ).

- La tendencia de la variabilidad es negativa y significativa $(-0.367, p=0.039)$ (Figura 3b). La tasa de descenso es $-0.21 \%$ / año. 
Tabla 1. Observatorio de Enguera ( $38^{\circ} 59^{\prime} \mathrm{N}, 0^{\circ} 41^{\circ} \mathrm{W}, 318 \mathrm{~m}$ s.n.m.)

\begin{tabular}{|c|c|c|c|c|c|c|c|c|c|c|c|}
\hline & $\mathrm{R}$ & AR & $|\mathrm{AR}|$ & $|\mathrm{ARr}|$ & & $\begin{array}{c}\mathrm{R} \\
\mathrm{m} . \mathrm{m} .\end{array}$ & & & $\begin{array}{c}|\mathrm{AR} r| \\
\mathrm{m} . \mathrm{m} .\end{array}$ & & \\
\hline (1) & (2) & (3) & (4) & (5) & (6) & (7) & (8) & (9) & $(10)$ & (11) & (12) \\
\hline 1950 & 369,5 & $-186,7$ & 186,7 & 33,6 & & & & & & & \\
\hline 1951 & 681,5 & 125,3 & 125,3 & 22,5 & & & & & & & \\
\hline 1952 & 269,8 & $-286,4$ & 286,4 & 51,5 & & & & & & & \\
\hline 1953 & 786,3 & 230,1 & 230,1 & 41,4 & & & & & & & \\
\hline 1954 & 363,8 & $-192,4$ & 192,4 & 34,6 & 1 & 519,1 & 9 & 64 & 28,2 & 13 & 144 \\
\hline 1955 & 354,8 & $-201,4$ & 201,4 & 36,2 & 2 & 559,3 & 20 & 324 & 28,0 & 12 & 100 \\
\hline 1956 & 550,1 & $-6,1$ & 6,1 & 1,1 & 3 & 533,9 & 13 & 100 & 27,5 & 11 & 64 \\
\hline 1957 & 614,0 & 57,8 & 57,8 & 10,4 & 4 & 543,8 & 14 & 100 & 25,8 & 6 & 4 \\
\hline 1958 & 682,0 & 125,8 & 125,8 & 22,6 & 5 & 518,5 & 8 & 9 & 21,2 & 3 & 4 \\
\hline 1959 & 731,6 & 175,4 & 175,4 & 31,5 & 6 & 520,2 & 10 & 16 & 20,9 & 2 & 16 \\
\hline 1960 & 453,0 & $-103,2$ & 103,2 & 18,6 & 7 & 532,2 & 12 & 25 & 18,7 & 1 & 36 \\
\hline 1961 & 358,6 & $-197,6$ & 197,6 & 35,5 & 8 & 516,2 & 7 & 1 & 21,6 & 4 & 16 \\
\hline 1962 & 558,2 & 2,0 & 2,0 & 0,4 & 9 & 472,5 & 5 & 16 & 27,2 & 10 & 1 \\
\hline 1963 & 379,1 & $-177,1$ & 177,1 & 31,8 & 10 & 465,3 & 4 & 36 & 25,9 & 7 & 9 \\
\hline 1964 & 463,2 & $-93,0$ & 93,0 & 16,7 & 11 & 430,7 & 1 & 100 & 25,1 & 5 & 36 \\
\hline 1965 & 406,0 & $-150,2$ & 150,2 & 27,0 & 12 & 461,1 & 3 & 81 & 26,4 & 8 & 16 \\
\hline 1966 & 221,1 & $-335,1$ & 335,1 & 60,3 & 13 & 459,5 & 2 & 121 & 26,7 & 9 & 16 \\
\hline 1967 & 616,6 & 60,4 & 60,4 & 10,9 & 14 & 488,3 & 6 & 64 & 31,9 & 14 & 0 \\
\hline 1968 & 420,4 & $-135,8$ & 135,8 & 24,4 & 15 & 546,1 & 15 & 0 & 35,2 & 17 & 4 \\
\hline 1969 & 726,4 & 170,2 & 170,2 & 30,6 & 16 & 550,4 & 18 & 4 & 34,4 & 15 & 1 \\
\hline 1970 & 344,4 & $-211,8$ & 211,8 & 38,1 & 17 & 546,8 & 16 & 1 & 35,1 & 16 & 1 \\
\hline 1971 & 817,6 & 261,4 & 261,4 & 47,0 & 18 & 634,9 & 29 & 121 & 37,5 & 20 & 4 \\
\hline 1972 & 899,2 & 343,0 & 343,0 & 61,7 & 19 & 632,0 & 27 & 64 & 37,0 & 19 & 0 \\
\hline 1973 & 501,9 & $-54,3$ & 54,3 & 9,8 & 20 & 681,6 & 33 & 169 & 40,5 & 22 & 4 \\
\hline 1974 & 373,3 & $-182,9$ & 182,9 & 32,9 & 21 & 636,1 & 30 & 81 & 41,9 & 26 & 25 \\
\hline 1975 & 1014,4 & 458,2 & 458,2 & 82,4 & 22 & 633,3 & 28 & 36 & 42,4 & 27 & 25 \\
\hline 1976 & 590,7 & 34,5 & 34,5 & 6,2 & 23 & 627,2 & 26 & 9 & 41,3 & 25 & 4 \\
\hline 1977 & 866,2 & 310,0 & 310,0 & 55,7 & 24 & 555,1 & 19 & 25 & 40,5 & 23 & 1 \\
\hline 1978 & 317,1 & $-239,1$ & 239,1 & 43,0 & 25 & 641,9 & 32 & 49 & 54,0 & 32 & 49 \\
\hline 1979 & 319,1 & $-237,1$ & 237,1 & 42,6 & 26 & 627,1 & 25 & 1 & 56,6 & 33 & 49 \\
\hline 1980 & 763,1 & 206,9 & 206,9 & 37,2 & 27 & 571,7 & 22 & 25 & 48,3 & 30 & 9 \\
\hline 1981 & 250,0 & $-306,2$ & 306,2 & 55,1 & 28 & 547,5 & 17 & 121 & 51,3 & 31 & 9 \\
\hline 1982 & 1282,8 & 726,6 & 726,6 & 130,6 & 29 & 520,2 & 11 & 324 & 46,3 & 29 & 0 \\
\hline 1983 & 240,7 & $-315,5$ & 315,5 & 56,7 & 30 & 568,5 & 21 & 81 & 45,5 & 28 & 4 \\
\hline 1984 & 515,6 & $-40,6$ & 40,6 & 7,3 & 31 & 594,4 & 24 & 49 & 40,8 & 24 & 49 \\
\hline 1985 & 372,5 & $-183,7$ & 183,7 & 33,0 & 32 & 588,0 & 23 & 81 & 39,7 & 21 & 121 \\
\hline 1986 & 620,5 & 64,3 & 64,3 & 11,6 & 33 & 638,1 & 31 & 4 & 36,5 & 18 & 225 \\
\hline 1987 & 752,5 & 196,3 & 196,3 & 35,3 & & & Suma & 2302 & & Suma & 1046 \\
\hline 1988 & 551,8 & $-4,4$ & 4,4 & 0,8 & & & suma & 2302 & & Stumet & \\
\hline 1989 & 705,3 & 149,1 & 149,1 & 26,8 & & & & & & & \\
\hline 1990 & 701,4 & 145,2 & 145,2 & 26,1 & & & & & & & \\
\hline
\end{tabular}

$\mathrm{R}$ (col. 2) Hluvia del año en mm. AR (col. 3) valor de la anomalía según (R-R). $|\mathrm{AR}|$ (col.4) anomalía absoluta. $\mid \mathrm{ARr}$ (col. 5) anomalía relativizada según $[(A R . / \bar{R})]^{*} 100$. Col. 6 el número de orden del año. R.m.m. (col. 7) media móvil de la lluvia (lag 9), en mm. Col. $8 \mathrm{el}$ número de orden de cada valor de Hluvia anual calculada por la media móvil. Col. 9. (col.6-col.8) ${ }^{2}$, diferencia de órdenes elevada al cuadrado. $\mid A \bar{R}$ rl m.m., la media móvil de la anomalía, (col. 10). Col, 11 orden de la anomalía relativizada. Col. $12(\mathrm{col} .6-11)^{2}$.

La precipitación media anual $\bar{R}$ es $556.2 \mathrm{~mm}$. El valor del cocficiente de la tendencia del volumen es 0.616 y su estadístico $u\left(r_{s}\right)=3.484$; en la variabilidad es de 0.825 y su estadístico $u\left(r_{*}\right)=4.666$. 

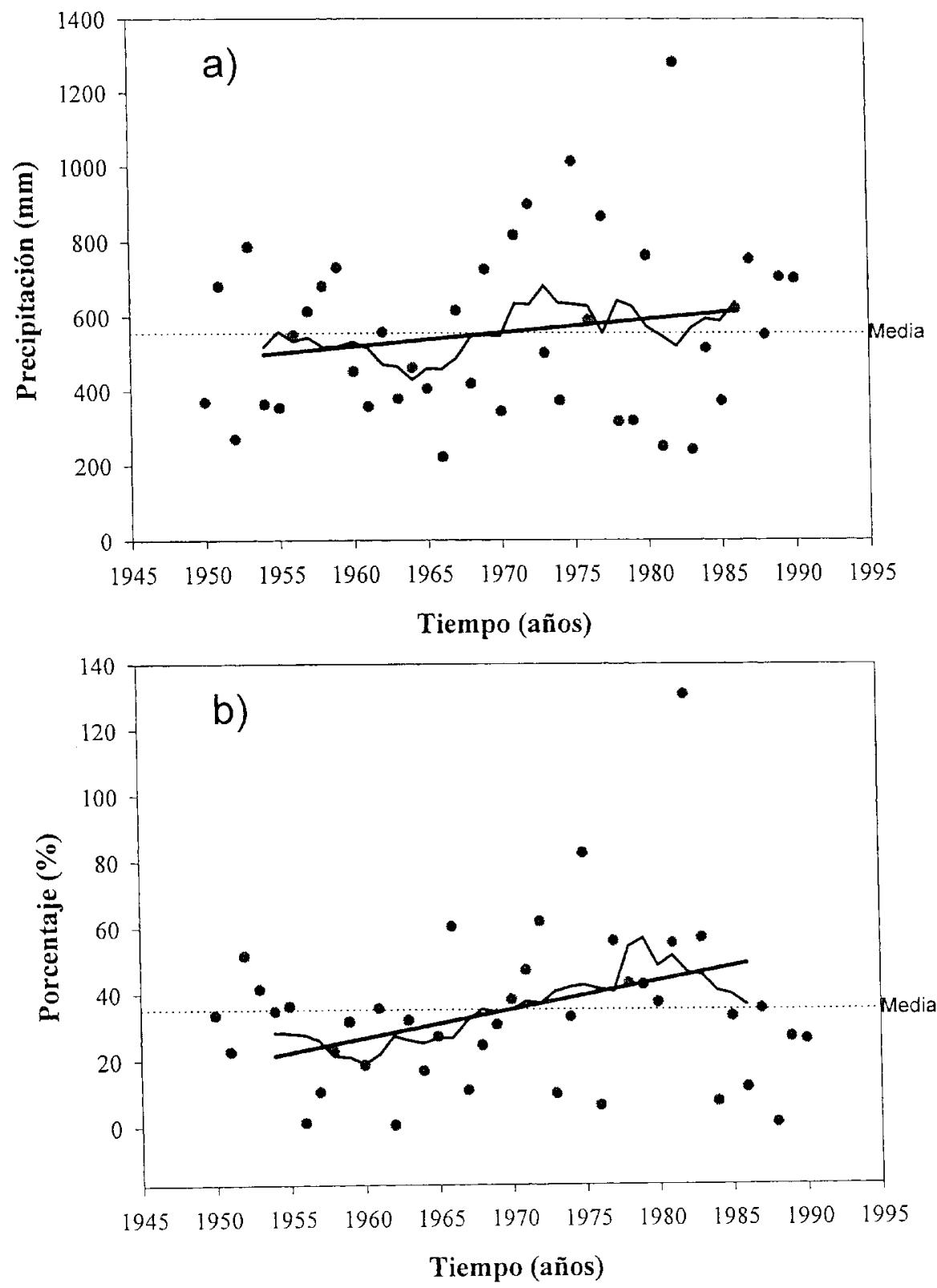

Figura 1. Observatorio de Enguera. (a) tendencia de la precipitación (volumen). (b) tendencia de la variabilidad expresada como anomalía absoluta sobre el valor de la media del período (en \%). En la figura se representan los valores absolutos anuales por medio de puntos, la media móvil con lapso de 9 años (trazo grueso), la media del período (trazo discontinuo) y la recta de tendencia. Más explicaciones en el texto 
Tabla 2. Observatorio de Adzeneta $\left(40^{\circ} 13^{\prime} \mathrm{N}, 1^{\circ} 10^{\prime} \mathrm{W}, 400 \mathrm{~m} \mathrm{s.n.m.)}\right.$

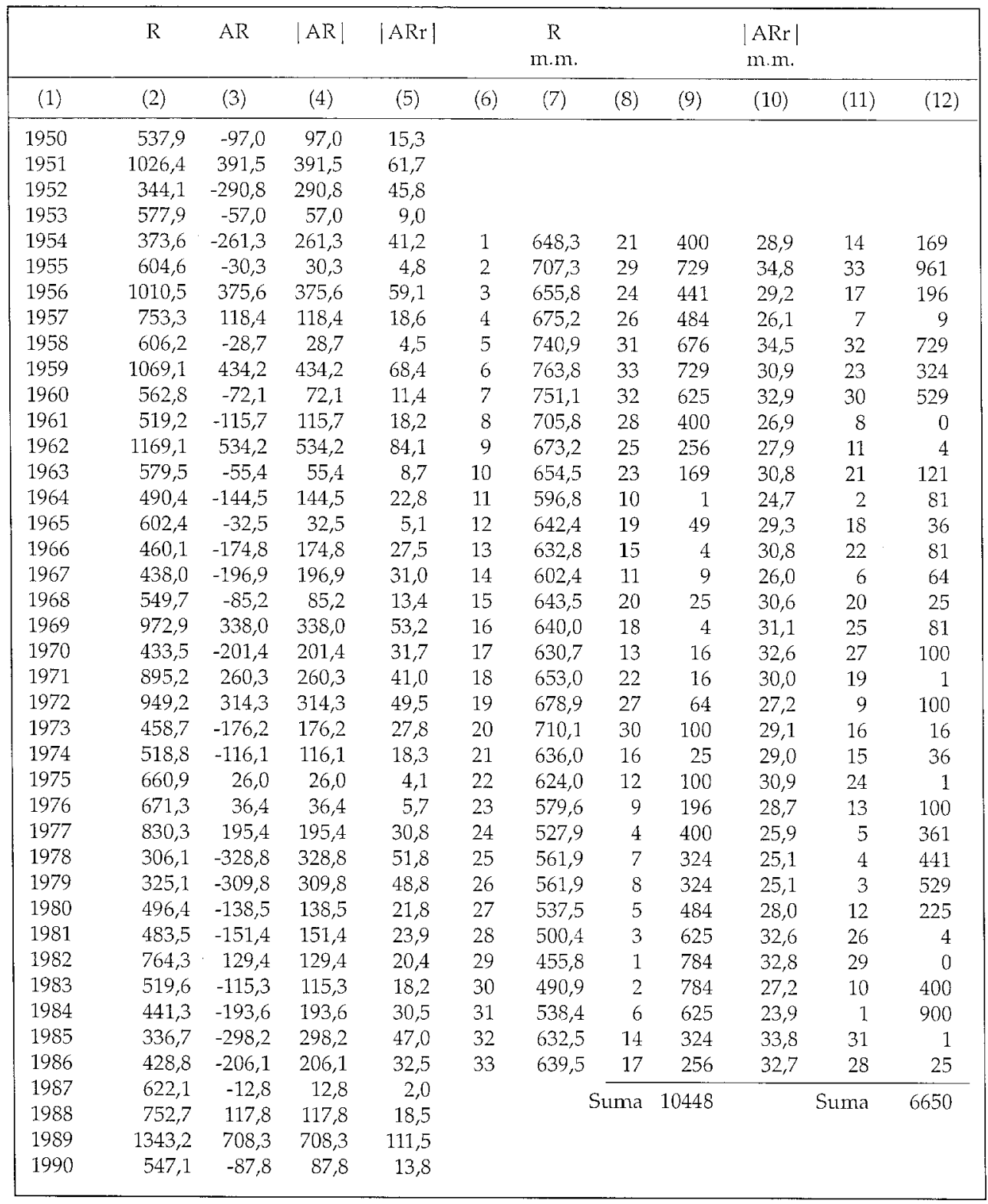

$R$ (col. 2) lluvia del año en mm. AR (col. 3) yalor de la anomalía según (R- $\bar{R})$. $|A R|$ (col.4) anomalía absoluta. $|A R r|$ (col. 5) anomalía relativizada según $[(A R . / \bar{R})]^{* 100 . ~ C o l . ~} 6$ el número de orden del año. R.m.m. (col. 7) media móvil de la lluvia (lag 9), en mm. Col. 8 el número de orden de cada valor de lluvia anual calculada por la media móvil. Col. 9. (col.6-col.8) , diferencia de órdenes elevada al cuadrado. $|\mathrm{ARr}| \mathrm{m} . \mathrm{m}$. la media móvil de la anomalía, (col. 10). Col. 11 orden de la anomalía relativizada. Col. 12 (col. $6-11)^{2}$.

La precipitación media anual $\overline{\mathrm{R}}$ es $634.9 \mathrm{~mm}$. El valor del coeficiente de la tendencia del volumen es -0.744 y su cstadístico $u\left(r_{i}\right)=4.209$; en la variabilidad es de -0.110 y su estadístico $u\left(r_{s}\right)=0.622$. 

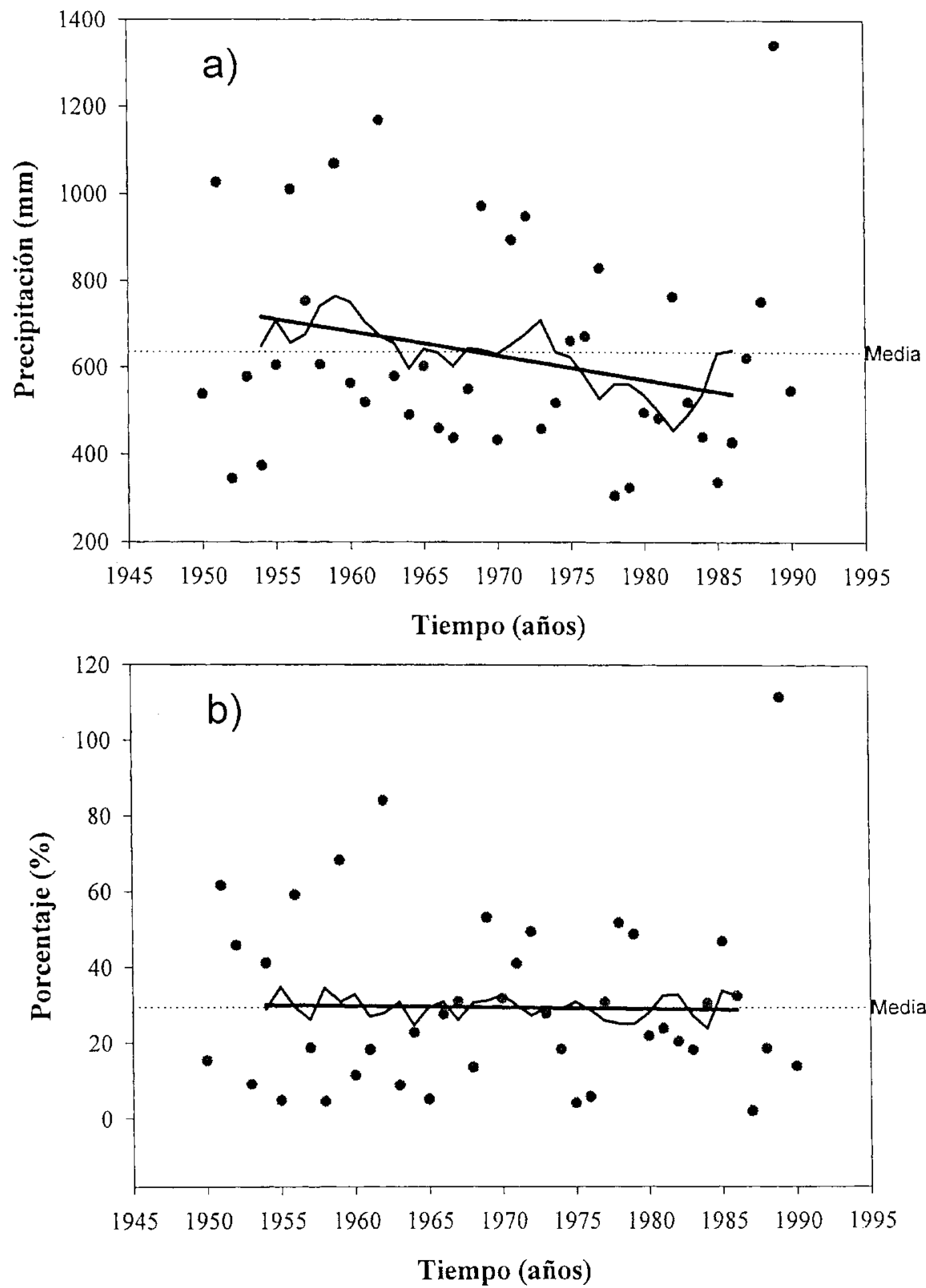

Figura 2. Observatorio de Adzeneta. (a) tendencia de la precipitación (volumen).

(b) tendencia de la variabilidad expresada como anomalía absoluta sobre el valor de la media del período (en \%). En la figura se representan los valores absolutos anuales por medio de puntos, la media móvil con lapso de 9 años (trazo grueso), la media del período (trazo discontinuo) y la recta de tendencia. Más explicaciones en el texto. 
Tabla 3. Observatorio de Vila-Real ( $39^{\circ} 56^{\prime} \mathrm{N}, 0^{\circ} 06^{\prime} \mathrm{W}, 42 \mathrm{~m} \mathrm{s.n.m.)}$

\begin{tabular}{|c|c|c|c|c|c|c|c|c|c|c|c|}
\hline & $\mathrm{R}$ & $\mathrm{AR}$ & $|\mathrm{AR}|$ & $|\mathrm{ARr}|$ & & $\begin{array}{c}\mathrm{R} \\
\mathrm{m} \cdot \mathrm{m} .\end{array}$ & & & $\begin{array}{c}|\mathrm{ARr}| \\
\mathrm{m.m} .\end{array}$ & & \\
\hline (1) & (2) & (3) & (4) & $(5)$ & (6) & (7) & (8) & $(9)$ & (10) & (11) & (12) \\
\hline 1950 & 247,8 & $-216,6$ & 216,6 & 46,6 & & & & & & & \\
\hline 1951 & 669,0 & 204,6 & 204,6 & 44,1 & & & & & & & \\
\hline 1952 & 109,3 & $-355,1$ & 355,1 & 76,5 & & & & & & & \\
\hline 1953 & 300,8 & $-163,6$ & 163,6 & 35,2 & & & & & & & \\
\hline 1954 & 259,7 & $-204,7$ & 204,7 & 44,1 & 1 & 406,6 & 4 & 9 & 40,6 & 32 & 961 \\
\hline 1955 & 307,2 & $-157,2$ & 157,2 & 33,8 & 2 & 464,4 & 18 & 256 & 42,7 & 33 & 961 \\
\hline 1956 & 730,5 & 266,1 & 266,1 & 57,3 & 3 & 436,0 & 11 & 64 & 39,0 & 31 & 784 \\
\hline 1957 & 582,0 & 117,6 & 117,6 & 25,3 & 4 & 460,7 & 17 & 169 & 33,7 & 25 & 441 \\
\hline 1958 & 452,9 & $-11,5$ & 11,5 & 2,5 & 5 & 501,4 & 27 & 484 & 34,6 & 28 & 529 \\
\hline 1959 & 768,3 & 303,9 & 303,9 & 65,4 & 6 & 519,9 & 30 & 576 & 30,6 & 18 & 144 \\
\hline 1960 & 413,5 & $-50,9$ & 50,9 & 11,0 & 7 & 518,8 & 29 & 484 & 30,9 & 19 & 144 \\
\hline 1961 & 331,3 & $-133,1$ & 133,1 & 28,7 & 8 & 478,2 & 23 & 225 & 26,9 & 11 & 9 \\
\hline 1962 & 666,8 & 202,4 & 202,4 & 43,6 & 9 & 457,6 & 16 & 49 & 25,7 & 7 & 4 \\
\hline 1963 & 426,4 & $-38,0$ & 38,0 & 8,2 & 10 & 437,3 & 12 & 4 & 30,1 & 16 & 36 \\
\hline 1964 & 297,6 & $-166,8$ & 166,8 & 35,9 & 11 & 400,2 & 2 & 81 & 23,5 & 3 & 64 \\
\hline 1965 & 364,6 & $-99,8$ & 99,8 & 21,5 & 12 & 423,2 & 7 & 25 & 26,0 & 9 & 9 \\
\hline 1966 & 397,4 & $-67,0$ & 67,0 & 14,4 & 13 & 424,2 & 8 & 25 & 25,8 & 8 & 25 \\
\hline 1967 & 270,2 & $-194,2$ & 194,2 & 41,8 & 14 & 445,6 & 15 & 1 & 30,4 & 17 & 9 \\
\hline 1968 & 434,3 & $-30,1$ & 30,1 & 6,5 & 15 & 470,1 & 21 & 36 & 33,9 & 26 & 121 \\
\hline 1969 & 620,6 & 156,2 & 156,2 & 33,6 & 16 & 468,5 & 20 & 16 & 34,2 & 27 & 121 \\
\hline 1970 & 339,9 & $-124,5$ & 124,5 & 26,8 & 17 & 472,6 & 22 & 25 & 33,3 & 22 & 25 \\
\hline 1971 & 859,1 & 394,7 & 394,7 & 85,0 & 18 & 482,0 & 24 & 36 & 32,2 & 20 & 4 \\
\hline 1972 & 647,2 & 182,8 & 182,8 & 39,4 & 19 & 506,9 & 28 & 81 & 28,2 & 13 & 36 \\
\hline 1973 & 283,4 & $-181,0$ & 181,0 & 39,0 & 20 & 532,5 & 31 & 121 & 32,3 & 21 & 1 \\
\hline 1974 & 401,5 & $-62,9$ & 62,9 & 13,5 & 21 & 492,8 & 26 & 25 & 33,3 & 23 & 4 \\
\hline 1975 & 482,0 & 17,6 & 17,6 & 3,8 & 22 & 491,3 & 25 & 9 & 33,7 & 24 & 4 \\
\hline 1976 & 494,5 & 30,1 & 30,1 & 6,5 & 23 & 438,5 & 13 & 100 & 26,2 & 10 & 169 \\
\hline 1977 & 664,0 & 199,6 & 199,6 & 43,0 & 24 & 404,9 & 3 & 441 & 24,6 & 6 & 324 \\
\hline 1978 & 264,0 & $-200,4$ & 200,4 & 43,2 & 25 & 444,4 & 14 & 121 & 24,5 & 5 & 400 \\
\hline 1979 & 326,0 & $-138,4$ & 138,4 & 29,8 & 26 & 433,0 & 10 & 256 & 26,9 & 12 & 196 \\
\hline 1980 & 384,0 & $-80,4$ & 80,4 & 17,3 & 27 & 422,4 & 6 & 441 & 28,4 & 14 & 169 \\
\hline 1981 & 344,9 & $-119,5$ & 119,5 & 25,7 & 28 & 415,4 & 5 & 529 & 28,4 & 15 & 169 \\
\hline 1982 & 639,0 & 174,6 & 174,6 & 37,6 & 29 & 395,7 & 1 & 784 & 24,2 & 4 & 625 \\
\hline 1983 & 299,0 & $-165,4$ & 165,4 & 35,6 & 30 & 430,1 & 9 & 441 & 22,0 & 1 & 841 \\
\hline 1984 & 386,0 & $-78,4$ & 78,4 & 16,9 & 31 & 464,5 & 19 & 144 & 22,8 & 2 & 841 \\
\hline 1985 & 432,0 & $-32,4$ & 32,4 & 7,0 & 32 & 550,7 & 33 & 1 & 37,5 & 29 & 9 \\
\hline 1986 & 486,0 & 21,6 & 21,6 & 4,7 & 33 & 544,3 & 32 & 1 & 38,9 & 30 & 9 \\
\hline 1987 & 574,0 & 109,6 & 109,6 & 23,6 & & \multirow{4}{*}{\multicolumn{2}{|c|}{ Suma }} & 6060 & & \multirow{4}{*}{ Suma } & \multirow[t]{4}{*}{8188} \\
\hline 1988 & 636,0 & 171,6 & 171,6 & 37,0 & & & & & & & \\
\hline 1989 & 1159,0 & 694,6 & 694,6 & 149,6 & & & & & & & \\
\hline 1990 & 288,0 & $-176,4$ & 176,4 & 38,0 & & & & & & & \\
\hline
\end{tabular}

$R$ (col. 2) lluvia del año en mm. AR (col. 3) valor de la anomalía según $(R-\bar{R})$. $|A R|(c o l .4)$ anomalía absoluta. $|A R r|$ (col. 5) anomalía relativizada según [(AR./R $)]^{*} 100$. Col. 6 el número de orden del año. R.m.m. (col. 7) media móvil de la lluvia (lag 9), en mm. Col. 8 el número de orden de cada valor de lluvia anual calculada por la media móvil. Col. 9. (col.6-col.8) ${ }^{2}$, diferencia de órdenes elevada al cuadrado. $|\mathrm{ARr}| \mathrm{m} . \mathrm{m}$. la media móvil de la anomalía, (col. 10). Col. 11 orden de la anomalía relativizada. Col. 12 (col. $6-11)^{2}$.

La precipitación media anual $\overline{\mathrm{R}}$ es $464.4 \mathrm{~mm}$. El valor del coeficiente de la tendencia del volumen es -0.012 y su estadístico $u\left(r_{s}\right)=0.068$; en la variabilidad es de -0.367 y su estadístico $u\left(r_{j}\right)=2.076$. 

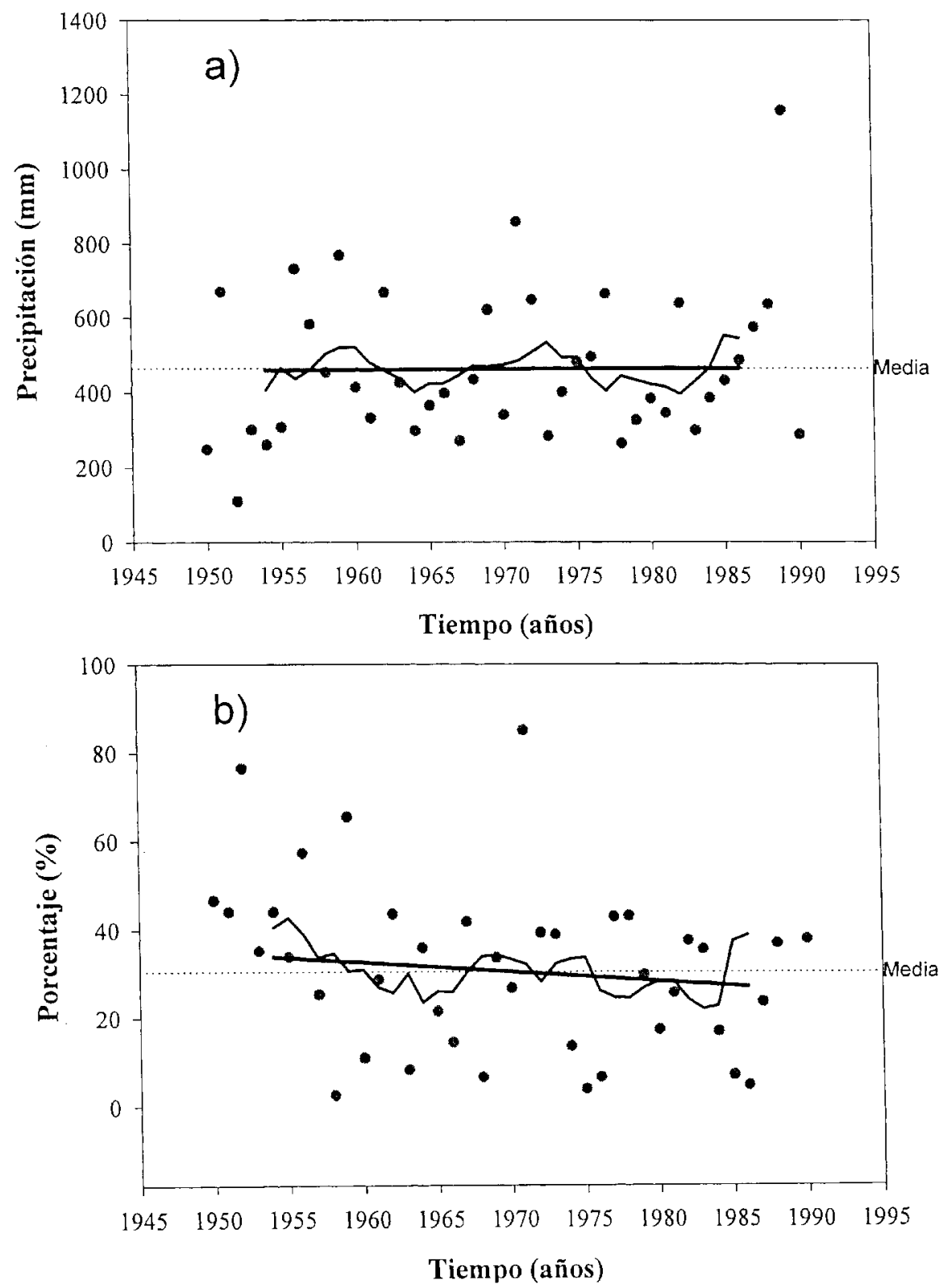

Figura 3. Observatorio de Vila-Real. (a) tendencia de la precipitación (volumen).

(b) tendencia de la variabilidad expresada como anomalía absoluta sobre el valor de la media del período (en \%). En la figura se representan los valores absolutos anuales por medio de puntos, la media móvil con lapso de 9 años (trazo grueso), la media del período (trazo discontinuo) y la recta de tendencia. Más explicaciones en el texto. 


\section{CONSIDERACIONES GENERALES SOBRE LOS RESULTADOS}

Las nueve combinaciones posibles entre el signo de la tendencia del volumen y el signo de la tendencia de su variabilidad representan los escenarios hipotéticos en que se puede mover el cambio pluvial, y por extensión cualquier variable del clima, en una localidad dada. Toda tendencia detectada en los valores anuales (positiva, negativa o nula) con tendencia de su variabilidad nula refleja una situación en la que la predicción alcanza una razonable fiabilidad. Otro tanto ocurre en donde la tendencia de la variabilidad descienda (se hace más precisa con el paso de los años) aunque las consecuencias variarán acorde la tendencia en los totales. Finalmente, la incertidumbre en las predicciones se incrementa cuando la variabilidad aumenta, con implicaciones diferentes según sea la tendencia del valor anual.

Se aclara que estos comentarios se realizan siempre a posteriori, es decir una vez analizada la serie de datos; por ello la hipótesis principal con la que se trabaja es que los cambios detectados se mantendrán de alguna manera en el futuro más inmediato. Este es uno de los puntos más débiles en todo análisis de tendencias climáticas, la asunción de que lo pasado se proyecta hacia el futuro.

Los tres casos descritos solamente son algunas de las nueve posibles combinaciones, pero ilustran el comportamiento más usual del conjunto de estaciones analizadas en la Comunidad Valenciana. Cada uno de ellos, caso de mantenerse las tendencias descritas, supone un escenario distinto para el futuro más inmediato con implicaciones en la gestión hidrológica, planificación forestal, manejo de sistemas naturales etc.

En el caso de Adzaneta las tendencias globales negativas, no afectadas de tendencia significativa en su anomalía, sugieren que cada vez los aportes serán menores y que el grado de fiabilidad de estas predicciones puede aceptarse con bastante confianza.

No ocurre lo mismo en el caso de Enguera, donde las tendencias positivas ocultan el fenómeno paralelo del aumento de su variabilidad, de modo que la fiabilidad de cualquier predicción se vería comprometida por la tendencia positiva en sus anomalías, es decir: la tendencia global surge de grandes variaciones en los totales de lluvia anual que aumentan progresivamente, lo que quiere decir que las cantidades anuales cada vez serán más contrastadas, esto es años con grandes aportes seguidos de períodos de escasez.

Por último el caso de Vila Real ilustra un comportamiento en el que a la ausencia de tendencias en los totales de lluvia corre parejo un descenso de su variabilidad, sugiriendo nuevamente una fiabilidad en sus predicciones. 


\section{DISCUSIÓN Y CONCLUSIONES}

El uso de técnicas sencillas debidamente combinadas para estudiar las tendencias de lluvia aporta una información relevante para conocer la estructura de su cambio en el tiempo. Entendemos que el signo de la tendencia y el valor del coeficiente de variación de la serie de datos no son suficientes para caracterizar el cambio del elemento pluvial. La variabilidad de la tendencia puede ocultar hechos relevantes que deberían considerarse en el proceso de planificación del recurso agua y en el análisis de las capacidades hidrológicas de un área.

La propuesta metodológica aquí desarrollada permite incorporar en los estudios de tendencias de lluvias el carácter dinámico que introduce la variabilidad entre años asociada a los valores absolutos. Así, independientemente del valor de tendencia observado (tanto de su significación como de su magnitud), el incremento de la variabilidad entre años detectada por el estudio de las series de anomalías de precipitación supondrá una mayor incertidumbre en el régimen de lluvias venidero y por lo tanto una mayor probabilidad de ocurrencia de años con anomalías extremas (graves sequías o inundaciones). A su vez, la observación de descensos en la variabilidad entre años implicaría una tendencia hacia un régimen de lluvias cada vez más homogéneo y predecible, y por lo tanto hacia un descenso en el riesgo de años extremos.

En nuestra opinión, ningún cálculo hidrológico de previsión de demandas futuras o excedentes debería realizarse sin considerar la variabilidad de la tendencia de las precipitaciones, tanto en las áreas y cuencas supuestamente receptoras, como sobre todo en las igualmente supuestas áreas y cuencas donantes. La variabilidad de cualquier tendencia puede inducir a errores de considerables consecuencias si no se considera en dichos cálculos.

En el momento presente la gestión del agua en España parece encaminada hacia la realización de trasvases entre cuencas; de los previstos en diversas situaciones destaca, por su magnitud y oposición social, el sistema de trasvases Ebro-Levante. Los autores, como ciudadanos, se preguntan si en los cálculos de estos movimientos de agua se ha tenido en cuenta la dinámica temporal de las precipitaciones aquí estudiada.

\section{ANEJO. EJEMPLOS DE CÁLCULO}

Los ejemplos presentados permiten seguir los pasos para calcular el signo de la tendencia y el signo de la variabilidad de la tendencia sobre valores reales de precipitaciones anuales de la Tabla 1. El modo de operar es el siguiente:

1. Se calcula el valor de la media de la precipitación de la serie. 
2. Se calcula la anomalía anual de precipitación, restando del valor de lluvia anual la media: $($ columna $2-$ media $)=$ columna 3 .

3. Se toma el valor absoluto de la anomalía eliminando los signos negativos (columna 4).

4. Se calcula la anomalía relativizada (columna $4 /$ media) $\times 100=$ columna 5 .

5. Se calcula la media móvil con lapso de 9 años de los volúmenes. El total de datos resultantes es de 33 años (columna 7).

6. Se asigna el valor del rango a la precipitación de cada año, con 1 la lluvia anual mínima (en media móvil) y 33 la lluvia anual de mayor cuantía (columna 8).

7. Se calcula la diferencia entre el número de orden del año y el valor del rango de los mm (columna 6 - columna 8), se eleva al cuadrado, (col. 9) y se suman los resultados.

8. Se calcula el valor del coeficiente de rangos $r_{s}$ y su estadístico según fórmulas precitadas.

9. Se procede desde el punto 4 de igual modo para calcular el coeficiente $r_{s}$ en las anomalías (columnas 10, 11 y 12).

Los valores de lluvia anual del observatorio de Enguera (Tabla 1), tienen un coeficiente de Spearman:

$$
r_{s}=1-\left(\frac{6 \times 2302}{33 \times\left(33^{2}-1\right)}\right)=0,616
$$

Cuyo estadístico estimado es

$$
u\left(r_{5}\right)=0,616 \sqrt{33-1}=3,484
$$

Para el $5 \%$ de probabilidad, las tablas estadísticas indican que el valor de la desviación normal $z$ es 1.96 en la prueba de dos colas. Se emplea este tipo de prueba debido a que la hipótesis nula es la existencia de diferencias. En consecuencia, como $3.484>1.96$ podemos concluir que hay una correlación significativa y por ello existe tendencia en los valores de lluvia.

El valor del coeficiente de las anomalías de los datos de la Tabla 1 es:

$$
r_{s}=1-\frac{6 \times 1046}{33 \times\left(33^{2}-1\right)}=0,825
$$

Cuyo estadístico estimado es 


$$
u\left(r_{s}\right)=0,825 \sqrt{33-1}=4,666
$$

con el mismo $5 \%$ de probabilidad, e igual valor de desviación normal z. En consecuencia, como $4.666>1.96$ podemos concluir que hay una correlación de rangos significativa y por ello existe una tendencia, de signo positivo además (el signo del coeficiente), en los valores de las anomalías de la lluvia.

Un método alternativo de cálculo de la significación, siempre que los pares de datos sean $>10$, es estimar la probabilidad con ayuda de la distribución de Student, según

$$
t=\left|r_{s}\right| \sqrt{\frac{N-2}{1 r_{s}^{2}}}
$$

con $N-2$ grados de libertad.

\section{AGRADECIMIENTOS}

Los autores manifiestan su agradecimiento al Dr. Pérez Cuevas (D. Geografía, U. Valencia) así como al Gobierno Regional de la Comunidad Valencia (Conselleria d'Ordenació del Territori, Generalitat Valenciana) por las facilidades dadas para acceder a las bases de datos empleadas en el presente estudio. Este trabajo ha sido financiado por CICYT, Proyecto CL195-1947-CO3-O3 y la Generalitat Valenciana, Projecte GV97-RN-14-2. Los autores agradecen asimismo la ayuda otorgada por el Instituto de Cultura Juan Gil-Albert (Alicante).

\section{BIBLIOGRAFÍA}

ANANTHAKRISHNAN, R. y SOMAN, M.K. (1989): «Statistical distribution of the daily rainfall and its association with the coefficient of variation of rainfall series». Int. J. Climatology, 9, 485-500.

BARROW, E.; HULME, M. y SEMENOV, M.(1996): «Effect of using different methods in the construction of climate change scenarios: Examples from Europe». Clim. Res., 7, 195-211

BRADLEY, R.S.; DIAZ, H.F.; EISCHEID, J.K.; JONES, P.D.; KELLY, P.M. y GOODESS, C.M. (1987): «Precipitation fluctuations over Northern Hemisphere land areas since the mid-19th century». Science, 237, 171-175.

CLARK, W.A.V. y HOSKING, P.L. (1986): Stntistical Methods for Geographers. Wiley, New York.

CORTE-REAL, J.; QIAN, B. y XU, H. (1998): «Regional climate change in Portugal: precipitation variability associated with large-scale atmospheric circulation». Int. J. Climatol., 18, 619-635. 
DAVIS, J.C. (1986): Statistics and data analysis in geology. John Wiley and Sons. New York. USA.

De LUIS, M.; GONZÁLEZ HIDALGO, J.C.; RAVENTÓS, J.; CORTINA, J. y SÁNCHEZ, J.R. (1998): «Estudio espacial y temporal de las tendencias de la lluvia en la Comunidad Valenciana (19611990)». Cuadernos de Investigación Geográfica, XXIV, 7-24.

DIAZ, H.F; BRADLEY, R.S. y EISCHEID, J.K. (1989): «Precipitation fluctuations over global land areas since the late 1800s». J. Geophys. Res., 94, D1, 1195-1210.

ESTEBAN-PARRA, M.J.; RODRIGO, F.S. y CASTRO-DIEZ, Y. (1995): «Temperature trends and change points in the northern Spain plateau during the last 100 years». Int. J. Climatol., 15, $1031-1042$.

FOLLAND, C.K.; PARKER, D.E. y KATES, F.E. (1984): «Worldwide marine temperature fluctuations 1856-1981». Nature, 310, 670-673.

FOWLER, A.M. y HENNESSY, K.J. (1995): «Potential impacts of global warming on the frequency and magnitude of heavy precipitation». Nat. Hazards, 11, 283-303.

GATES, W.L.; MITCHELL, J.F.B.; BOER, G.J.; CUBASCH, U. y MELESHKO, V.P. (1992): In: HOUGHTIN, J.T., CALLANDER, B.A. y VARNEY, S.K. Climatic change 1992. The supplementary report to the IPCC Scientific Assessment. Cambridge University Press. Cambridge. 97-134

GORDEN, H.B.; WHETTON, P.H.; PITTOCK, A.B.; FOWLER, A.M. y HAYLOCK, M.R. (1992): «Simulated changes in daily rainfall intensity due to the enhanced greenhouse effect: implications for extreme rainfall events». Clim. Dyn., (7-8) 83-102

GREGORY, J.M. y MITCHELL, J.F.B. (1995): «Simulation of daily variability of surface temperature and precipitation over Europe in the current and $2 \times \mathrm{CO}_{2}$ climates using the UKMO climate model». Meteorol. Soc., 121, 1451-1476

GREGORY, S. (1978): Statistical Methods and the Geographer. Longman. New York.

GROISMAN, P. y LEGATES, D.R. (1994): «The accuracy of United States precipitation data». Bulll. Amer. Meteorol. Soc., 75, 215-227.

HENNESSY, K.J.; GREGORY, J.M. y MITCHELL, J.F.B. (1997): «Changes in daily precipitation under enhanced greenhouse conditions». Clim. Dyn., 13, 667-680

HOUGHTON, J.T.; JENKINS, G.J. y EPHRAUMS, J.J. (eds) (1990): Climate Change: The IPCC Scientific Assessment. Cambridge University Press. Cambridge.

HOUGHTON, J.T.; MEIRA FILHO, L.G.; CALLANDER, B.A.; HARRIS, N.; KATTENBERG, A. y MASKELL, K. (eds) (1996): Climate Change 1995. The Science of Climate Change. Cambridge University Press, UK, $572 \mathrm{p}$.

HULME, M. (1992): «Rainfall changes in Africa: 1931-1960 to 1961-1990». Int. J. Climntol., 12, 685-699. 
HULME, M. (1995): «Estimating global changes in precipitation». Weather, 50, 34-42.

HULME, M. y NEW, M. (1997): «Dependence of large-scale precipitation climatologies on temporal and spatial sampling». J. Climate, 10, 1099-1113.

JONES, P.D.; WIGLEY, T.M.L. y WRIGHT, P.B. (1986): «Global temperature variations between 1861 and 1984». Nature, 322, 430-434

KATZ, R.W. y BROWN, B.G. (1992): «Extreme events in a changing climate. Variability is more important than averages». Climatic Change, 21, 289-302.

KEIM, B.D.; FAIERS, G.E.; MULLER, R.A.; GRYMES III, J.M. y ROHLI, R.V. (1995): «Long-term trends of precipitation and runoff in Lousiana, USA». Int. J. Climatol., 15, 531-541.

KRAUS, E.B. (1977): «Subtropical droughts and cross-equatorial transports». Mon. Wen. Rev., 105, 1009-1018.

KUTIEL, H. y MAHERAS, P. (1998): «Variations in the temperature regime across the Mediterranean during the last century and their relationship with circulation indices». Theoretical \& Applied Climatology, 61, 39-53.

KUTIEL, H. y MAHERAS, P. (1998): «Variations in the temperature regime across the Mediterranean during the last century and their relationship with circulation indices». Theoretical \& Applied Climatology, 61, 39-53.

LABAJO, J.L.; PIORNO, A. e IZQUIERDO, M.J. (1998): «Temporal behaviour of the annual mean pressure on the northern Spanish plateau between 1945 and 1994». Int. J. Climatol., 18, 637-647.

LANZANTE, J.R. (1996): «Resistant, robust and non-parametric techniques for the analysis of climate data: theory and examples including applications to historical radiosonde station data». Int. J. Climatol., 16, 1197-1226.

LOUREIRO, N.S y COUTINHO, M.A. (1995): «Rainfall changes and rainfall erosivity increase in the Algarve (Portugal)». Catena, 24, 55-67.

MAHERAS, P. (1988): «Changes in precipitation conditions in the Western Mediterranean over the last century». J. Climate, 8, 179-189

MAHERAS, P. y KOLYVA-MACHERA, F. (1990): «Temporal and spatial characteristics of annual precipitation over the Balkans in the Twentieth Century». Int. J. Climatol., 10, 495-504.

McGUFFIE, K.; HENDERSON-SELLER, A.; HOLBROOK, N.; KOTHAVALA, Z.; BALACHOVA, O. y HOEKSTRA, J. (1999): «Assessing simulations of daily temperature and precipitation variability with global climate models for present and enhanced greenhouse climates». Int. J. Climatol., $19,1-26$.

MEARNS, L.O.; ROSENZWEIG, C. y GOLDBERG, R. (1996): «The effect of changes in daily and interannual climatic variability on CERES-wheat: A sensitivity study». Climatic Change, 32, 257-292. 
MEARNS, L.O.; Rosenzweig, C. y Goldberg, R. (1997): «Mean and variance change in climate scenarios: Methods, agricultural applications, and measures of uncertainty». Climatic Change, 35, 367-396

MENDOÇA LEITE, S. y PEIXOTO, J.P. (1996): «The autoregresive model of climatological time series: an application to the longest time series in Portugal». Int. J. Climatol., 16, 1165-1173.

MORON, V.; BIGOT, S. y ROUCOU, P. (1995): «Rainfall variability in subequatorial America and Africa and relationship with the main sea-surface temperature modes». Int. J. Climatol., 15, 1297-1322.

ONATE, J.J. y POU, A. (1996): «Temperature variations in Spain since 1901: a preliminary analysis». Int. J. Climatol., 16, 805-815

OSBORN, T.J. y HULME, M. (1998): «Evaluation of the European daily precipitation characteristics from the atmospheric model Intercomparison Project». Int. J. Climatol., 18, 505-522.

PALUTIKOF, J.P; GOODESS, C.M. y HACOT, H. (1996): «Future rainfall trends over the mediterranean». Book of abstracts. International conference on mediterranean desertification. Crete-Hellas, 29 Oct - 1 Nov 1996.

PÉREZ-CUEVA, A. (Dir) (1994): Atlas climàtic de la Comunitat Valenciana. Direc. General d'Urbanisme i Ordenació del Territori, Generalitat Valenciana, Col.leció Territori, No 4.

REED, D.N. (1986): «Simulation of time series of temperature and precipitation over eastern England by an atmospheric general circulation model». J. Climatol., 6, 233-253

RIND, D.; GOLDBERG, R. y RUEDY, R. (1989): «Change in climate variability in the $21^{\text {st }}$ century». Climatic Change, 14, 5-37.

RODRIGUEZ-PUEBLA, C.; ENCINAS, A.H.; NIETO, S. y GARMENDIA, J. (1998): «Spatial and temporal patterns of annual precipitation variability over the Iberian Peninsula». Int.J. Climatol., 18, 299-316.

ROSSI, R.; MULLA, D.; JOURNEL, A. y FRANZ, E. (1992): «Geostatistical tools for modeling and interpreting ecological spatial dependence». Ecological Monographs, 62-2, 277-314.

SALINGER, M.J.; BASHER, R.E.; FITZHARRIS, B.B.; HAY, J.E.; JONES, P.D.; MaCVEIGH, J.P. y SCHMIDELY-LELEU, I. (1995): "Climate trends in the south-west pacific». Int. J. Climatol., 15, 285-302.

SEMENOV, M.A. y PORTER, J.R. (1994): «The implications and importance of non-linear responses in modelling og growth and development of wheat». In: GRASMAN, J. and van STRATEN, G. (Eds.) Predictability and non-linear modelling in natural sciences and economics. Pudoc. Wageningen.

SNEYERS, R. (1990): «On the Statistical Analysis of Series of Observations, WMO Technical Note (143)» World Meteorological Organization. Geneva, $192 \mathrm{p}$.

SNEYERS, R. (1992): «Use and misuse of statistical methods for detection of climatic change». En Climate Change Detection Project, Report on the informal Planning Meeting on Statistical Procedures for Climate Change Detection. WCDMP, (20) WMO. 
SRIKANTAN, R. y STEWART, B.J. (1991): "Analysis of Australian reinfall data with respect to climate variability and change». Anst. Meteorol. Mngnzine, 39, 11-20.

SUPPIAH, R. y HENNESSY, K.J. (1998): «Trends in total rainfall, heavy rain events and number of dry days in Australia, 1910-1990». Int. J. Climatol., 10, 1141-1164.

TÜRKES, M. (1996): «Spatial and temporal analysis of annual rainfall variations in Turkey». Int. J. Climatol., 16, 1057-1076.

WAYLEN, P.R., QUESADA, M.E. y CAVIEDES, C.N. (1996): «Temporal and spatial variability of annual precipitation in Costa Rica and the Southern Oscillation". Int. J. Climatol., 16, 173-193.

WHEELER, D. y MARTIN-VIDE, J. (1992): «Rainfall characteristics of mainland Europe's most southerly stations». Int. J. Climatol., 12, 69-76.

WHETTON, P.H.; FOWLER, A.M.; HAYLOCK, M.R. y PITTOCK, A.B. (1993): «Implications of climate change due to the enhanced greenhouse effect on floods and droughts in Australia». Climatic Clange, 25, 289-317.

WILSON, C.A. y MITCHELL, J.F.B. (1987): «Simulated climate and $\mathrm{CO}_{2}$-induced climate change over western Europe». Climatic Change, 10, 11-42.

YU, B. y NEIL, D.T. (1993): «Long-term variations in regional rainfall in the south-west of western Australia and the difference between average and high intensity rainfalls». Int. J. Climntol., 13, 77-88. 